\title{
Aberrant expression of nuclear HDAC3 and cytoplasmic CDH1 predict a poor prognosis for patients with pancreatic cancer
}

\author{
Feng Jiao ${ }^{1,2 *}$, Hai Hu${ }^{1 *}$, Ting Han ${ }^{1,2 *}$, Meng Zhuo ${ }^{1 *}$, Cuncun Yuan ${ }^{3}$, Haiyan Yang ${ }^{1}$, \\ Lei Wang ${ }^{1,2}$, Liwei Wang ${ }^{1,2}$ \\ ${ }^{1}$ Department of Medical Oncology and Pancreatic Cancer Center, Shanghai General Hospital, Shanghai Jiao Tong University \\ School of Medicine, Shanghai 201620, China \\ ${ }^{2}$ Shanghai Key Laboratory of Pancreatic Diseases, Shanghai 201620, China \\ ${ }^{3}$ Department of Pathology, Shanghai General Hospital, Shanghai Jiao Tong University School of Medicine, Shanghai 201620, \\ China \\ *These authors are contributed equally to this work
}

Correspondence to: Feng Jiao, e-mail: jiao_f@outlook.com

Lei Wang, e-mail: wang_lei744@hotmail.com

Liwei Wang, e-mail: yzwlw@hotmail.com

Keywords: pancreatic cancer, histone deacetylases 3, CDH1, subcellular localization, prognosis

Received: January 05, 2016

Accepted: February 11, 2016

Published: February 24, 2016

\section{ABSTRACT}

Previous studies showed that aberrant CDH1 or/and HDAC3 localization is essential for the progression of some human cancers. Here, we investigate the prognostic significance of aberrant CDH1 and HDAC3 localization in 84 pancreatic cancer patients. Our results show that increases in both membrane and cytoplasmic CDH1 correlate with lymph node metastasis $(P=0.026$ and $P<0.001$, respectively) and clinical stage $(P=0.020$ and $P<0.001$, respectively). Increased nuclear HDAC3 correlates with lymph node metastasis $(P<0.001)$ and advanced clinical stage $(P<0.001)$, but increased cytoplasmic HDAC3 does not $(P>0.05)$. Multivariate analysis showed that nuclear HDAC3 and cytoplasmic CDH1 $(P=0.001$ and $P=0.010$, respectively $)$, as well as tumor differentiation $(P=0.009)$ are independent prognostic factors. Most importantly, patients with high co-expression of nuclear HDAC3 and cytoplasmic CDH1 had shorter survival times $(P<0.001)$, more frequent lymph node metastasis $(P<0.001)$, and advanced clinical stage $(P<0.001)$. Our studies provide convincing evidence that nuclear HDAC3 and cytoplasmic CDH1 have independent prognostic value in pancreatic cancer and provide novel targets for prognostic therapeutics.

\section{INTRODUCTION}

Pancreatic cancer (PC) is one of the most aggressive and lethal malignancies, causing the deaths of an estimated 330,400 men and women worldwide in 2012 [1]. Total deaths due to PC are projected to increase dramatically, making it second leading cause of cancer-related deaths in the United States by 2030 [2]. Gemcitabine, the current standard first-line treatment, offers marginal symptom control and prolongation of life. Clinical trials aiming to improve the efficacy of gemcitabine have provided little improvement in survival outcomes [3]. New therapeutic strategies, including therapeutic antibodies or/and small molecule inhibitors, have been successful for a number of malignancies, but results obtained on PC treatments have so far been extremely frustrating [4]. A number of molecular mechanisms responsible for transformation and progression of $\mathrm{PC}$ have been identified, providing a set of potential pharmacological targets [5]. Among these is loss of adhesion between tumor cells caused by downregulation of CDH1 (also called E-cadherin) in response to genetic or epigenetic changes [6-8].

Histone acetylation is a dynamic epigenetic mechanism regulated by the histone acetyltransferases (HAT) and histone deacetylases (HDACs). HDAC3 (histone deacetylases 3), a member of class I HDACs, is overexpressed in the majority of carcinomas [9, 10], and is one of the most frequently upregulated genes 
in cancer [11]. Our previous study shows increased HDAC3 expression in PC [12]. HDAC3 could function as an oncogenic protein, promoting PC cell proliferation, migration, and invasion, as well as increasing drug resistance [12]. HDAC3 inversely correlates with CDH1 expression in ovarian carcinoma, and HDAC3 siRNA knock down in ovarian carcinoma cells reduced cell migration and increased CDH1 expression [13]. HDAC3 represses CDH1 through interactions with epithelialmesenchymal transition (EMT) regulators including Snail and Twist1 [14].

This study uses high-throughput tissue microarray (TMA) and immunohistochemistry to investigate the expression and subcellular localization of $\mathrm{CDH} 1$ and HDAC3 in PC tissues. We analyze their association with clinicopathological factors, and address their possible value as prognostic indicators.

\section{RESULTS}

\section{Expression of CDH1 and HDAC3 in PC tissues and adjacent normal tissues}

Immunohistochemistry results are summarized in Tables 1 and 2. Strong membrane localization of CDH1 was observed in $85.7 \%(72 / 84)$ of normal tissues adjacent to PC (Figure 1A). In contrast, cell membrane expression of CDH1 was greatly reduced in PC tissues (Figure 1B), with high expression in $63.1 \%(53 / 84)$ of cases. Interestingly, higher cytoplasmic $\mathrm{CDH} 1$ expression was observed in PC samples (Figure 1C); 33.3\% of tumor samples (28/84) but only $11.9 \%$ (10/84) of adjacent tissue samples displayed high cytoplasmic CDH1.

HDAC3 was distributed in the cytoplasm and nucleus. As shown in Figure 1D, nuclear HDAC3 was highly expressed in $54.8 \%(46 / 84)$ of PC tissues. In contrast, HDAC3 was only seen in the nucleus of $19 \%$ $(16 / 84)$ of noncancerous tissues (Figure 1E). There was no difference in cytoplasmic HDAC3 expression between PC tissues and noncancerous samples (54.8\%, 46/84 vs. $58.3 \%, 49 / 84$; Figure 1E, 1F).

\section{Correlations of CDH1 and HDAC3 expression in PC tissues}

An inverse correlation was identified between low membrane expression of CDH1 and high nuclear HDAC3 expression (Spearman correlation coefficient $r=-0.348$, $P=0.001$, Supplementary Table S1). High cytoplasmic CDH1 expression positively correlated with high nuclear HDAC3 expression (Spearman correlation coefficient $r=0.440, P<0.001$, Table 3 ). No correlations were found between cytoplasmic HDAC3 expression and CDH1 expression location $(P>0.05$, Supplementary Tables S2, S3).

\section{Relationship of clinicopathological features with CDH1 and HDAC3 expression in PC patients}

The relationships of $\mathrm{CDH} 1$ and $\mathrm{HDAC} 3$ expression levels with clinicopathological features of PC were evaluated by immunohistochemistry. As summarized in Table 4, CDH1 cell membrane expression correlated with lymph node metastasis $(P=0.026)$ and clinical stage $(P=0.020)$. High cytoplasmic CDH1 strongly correlated with lymph node metastasis (N classification, $P<0.001)$ and advanced clinical stage $(P<0.001)$. Neither cytoplasmic nor membrane CDH1 were associated with patients' gender, age, tumor location, tumor size, tumor differentiation, invasion depth, distant metastasis, abdominal pain, jaundice or nervous invasion $(P>0.05)$.

As summarized in Table 5, no correlations were observed between cytoplasmic levels of HDAC3 and patients' clinicopathologic features. Nuclear HDAC3 staining correlated with lymph node metastasis $(P<0.001)$ and clinical stage $(P<0.001)$, but did not correlate with patient's gender, age, tumor location, tumor size, tumor differentiation, invasion depth, distant metastasis, abdominal pain, jaundice, or nervous invasion $(P>0.05)$.

\section{Associations between CDH1 and HDAC3 expression and survival}

Kaplan-Meier analysis and log-rank test were used to investigate the prognostic value of CDH1 and HDAC3 expression and classic clinicopathologic characteristics on patient survival. In univariate analysis, both membrane and cytoplasmic CDH1 expression, as well as nuclear HDAC3, were closely associated with overall survival (OS) of PC patients $(P=0.012, P<0.001$, and $P<0.001$, respectively; Table 6), with Spearman correlation coefficients of $0.240,-0.435$, and -0.530 (Supplementary Table S4), respectively. The log-rank test results showed that the aberrant expression levels of these proteins correlated strongly with poorer survival in PC patients $(P<0.001$; Figure 2). As shown in Table 7, the cumulative 1 -year survival rate was $58 \%$ in the high membrane CDH1 group (95\% confidence interval [CI], 0.443-0.717), whereas it was only $32 \%(95 \% \mathrm{CI}, 0.163-0.477)$ in the low expression group (Figure 2A). The cumulative 1-year survival rate was $63 \%(95 \% \mathrm{CI}, 0.512-0.748)$ in the low cytoplasmic CDH1 group, whereas it was only $21 \%$ (95\% CI, 0.053-0.367) in the high-expression group (Figure 2B). The 1-year survival rate was 79\% in the low nuclear HDAC3 group (95\% CI, 0.653-0.927), whereas it was only $24 \%(95 \% \mathrm{CI}, 0.122-0.358)$ in the high staining group (Figure 2C). There was no difference in survival time associated with cytoplasmic HDAC3 expression (low vs. high, 47\% (95\% CI, 0.313-0.627) vs. 50\% (95\% CI, 0.363-0.637); Figure 2D). 
Table 1: Comparisons with CDH1 expression between $\mathrm{PC}$ and paired adjacent normal tissues $(n=84)$

\begin{tabular}{|c|c|c|c|c|c|c|c|}
\hline \multirow{2}{*}{ Tissue sample } & \multirow{2}{*}{ No.of patients } & \multicolumn{2}{|c|}{ Membrane CDH1 $(n, \%)$} & \multirow{2}{*}{$P$-value } & \multicolumn{2}{|c|}{ Cytoplasmic CDH1 $(n, \%)$} & \multirow{2}{*}{$P$-value } \\
\hline & & Low & High & & Low & High & \\
\hline Tumor & 84 & $31(36.9)$ & $53(63.1)$ & \multirow[b]{2}{*}{$0.001 *$} & $56(66.7)$ & $28(33.3)$ & \multirow[b]{2}{*}{$0.001 *$} \\
\hline $\begin{array}{c}\text { Adjacent } \\
\text { normal }\end{array}$ & 84 & $12(14.3)$ & $72(85.7)$ & & $74(88.1)$ & $10(11.9)$ & \\
\hline
\end{tabular}

Table 2: Comparisons with HDAC3 expression between PC and paired adjacent normal tissues $(n=84)$

\begin{tabular}{|c|c|c|c|c|c|c|c|}
\hline \multirow{2}{*}{ Tissue sample } & \multirow{2}{*}{ No.of patients } & \multicolumn{2}{|c|}{ Nuclear HDAC3 $(n, \%)$} & \multirow{2}{*}{$P$-value } & \multicolumn{2}{|c|}{ Cytoplasmic HDAC3 ( $n, \%)$} & \multirow{2}{*}{$P$-value } \\
\hline & & Low & High & & Low & High & \\
\hline Tumor & 84 & $38(45.2)$ & $46(54.8)$ & \multirow{2}{*}{$<0.001^{*}$} & $38(45.2)$ & $46(54.8)$ & \multirow{2}{*}{0.641} \\
\hline Adjacent normal & 84 & $68(81.0)$ & $16(19.0)$ & & $35(41.7)$ & $49(58.3)$ & \\
\hline
\end{tabular}

Table 3: Association between nuclear HDAC3 and cytoplasmic CDH1 expression

\begin{tabular}{|c|c|c|c|c|}
\hline \multirow{2}{*}{ Tumor tissue sample } & \multicolumn{2}{c}{ Nuclear HDAC3 } & \multirow{2}{*}{ Correlation coefficient } & \multirow{2}{*}{ P-value } \\
\cline { 2 - 3 } Cytoplasmic CDH1 Low & 34 & 22 & 0.440 & $<0.001^{*}$ \\
\hline Cytoplasmic CDH1 High & 4 & 24 & & $<$ \\
\hline
\end{tabular}
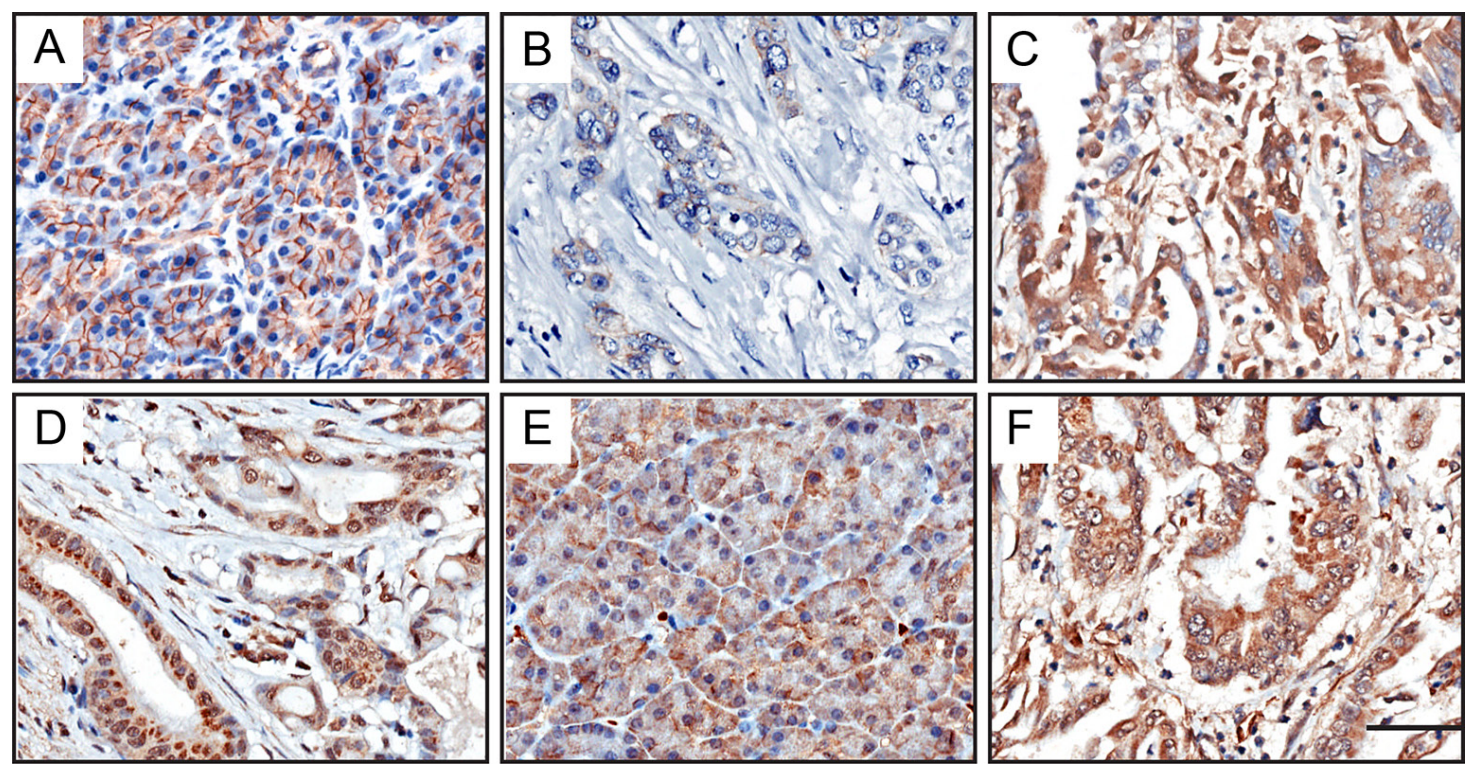

Figure 1: Immunohistochemical expression levels and localization of CDH1 and HDAC3 in PC tissues. Strong membraneassociated CDH1 was observed in adjacent normal tissues (A). Low membrane CDH1 (B) and high cytoplasmic CDH1 (C) was found in tumor cells. Higher level of nuclear HDAC3 was observed in PC tissues (D), than in adjacent normal tissues (E). There was no difference in cytoplasmic HDAC3 expression between PC tissues and noncancerous samples (E, normal tissue; (F), tumor tissue). Scale bar, $50 \mu \mathrm{m}$. 
Table 4: Correlation between the clinicopathologic characteristics and CDH1 expression $(n=84)$

\begin{tabular}{|c|c|c|c|c|c|c|c|}
\hline \multirow{2}{*}{$\begin{array}{c}\text { Clinicopathological } \\
\text { parameters }\end{array}$} & \multirow{2}{*}{ No.of patients } & \multicolumn{3}{|c|}{ Membrane CDH1 $(n, \%)$} & \multicolumn{3}{|c|}{ Cytoplasmic CDH1 $(n, \%)$} \\
\hline & & Low & High & $P$-value & Low & High & $P$-value \\
\hline Cases & 84 & $31(36.9)$ & $53(63.1)$ & & $56(66.7)$ & $28(33.3)$ & \\
\hline \multicolumn{8}{|l|}{ Age (years) } \\
\hline$\leq 60$ & 39 & $17(43.6)$ & $22(56.4)$ & \multirow{2}{*}{$0.237^{\mathrm{a}}$} & $26(66.7)$ & $13(33.3)$ & \multirow{2}{*}{$1.000^{\mathrm{a}}$} \\
\hline$>60$ & 45 & $14(31.1)$ & $31(68.9)$ & & $30(66.7)$ & $15(33.3)$ & \\
\hline \multicolumn{8}{|l|}{ Gender } \\
\hline Male & 51 & $21(41.2)$ & $30(58.8)$ & \multirow{2}{*}{$0.313^{\mathrm{a}}$} & $34(66.7)$ & $17(33.3)$ & \multirow{2}{*}{$1.000^{\mathrm{a}}$} \\
\hline Female & 33 & $10(30.3)$ & $23(69.7)$ & & $22(33.3)$ & $11(33.3)$ & \\
\hline \multicolumn{8}{|l|}{ Tumor location } \\
\hline Head, neck & 56 & $24(42.9)$ & $32(57.1)$ & \multirow{2}{*}{$0.110^{\mathrm{a}}$} & $35(62.5)$ & $21(37.5)$ & \multirow{2}{*}{$0.252^{\mathrm{a}}$} \\
\hline Body, tail & 28 & $7(25.0)$ & $21(75.0)$ & & $21(75.0)$ & $7(25.0)$ & \\
\hline \multicolumn{8}{|l|}{ Tumor size $(\mathrm{cm})$} \\
\hline$\leq 3$ & 25 & $9(36.0)$ & $16(64.0)$ & \multirow{2}{*}{$0.911^{\mathrm{a}}$} & $18(72.0)$ & $7(28.0)$ & \multirow{2}{*}{$0.500^{\mathrm{a}}$} \\
\hline$>3$ & 59 & $22(37.3)$ & $37(62.7)$ & & $38(64.4)$ & $21(35.6)$ & \\
\hline \multicolumn{8}{|l|}{ Tumor differentiation } \\
\hline Well, moderate & 57 & $21(36.8)$ & $36(63.2)$ & \multirow{2}{*}{$0.986^{\mathrm{a}}$} & $40(70.2)$ & $17(29.8)$ & \multirow{2}{*}{$0.322^{\mathrm{a}}$} \\
\hline Poor & 27 & $10(37.0)$ & $17(63.0)$ & & $16(59.3)$ & $11(40.7)$ & \\
\hline \multicolumn{8}{|l|}{ Invasion depth } \\
\hline $\mathrm{T} 1+\mathrm{T} 2$ & 71 & $27(38.0)$ & $44(62.0)$ & \multirow{2}{*}{$0.618^{\mathrm{a}}$} & $49(69.0)$ & $22(31.0)$ & \multirow{2}{*}{$0.286^{\mathrm{a}}$} \\
\hline $\mathrm{T} 3+\mathrm{T} 4$ & 13 & $4(30.8)$ & $9(69.2)$ & & $7(53.8)$ & $6(46.2)$ & \\
\hline \multicolumn{8}{|l|}{ Lymph nodes metastasis } \\
\hline N0 (negative) & 51 & $14(27.5)$ & $37(72.5)$ & \multirow{2}{*}{$0.026^{a^{*}}$} & $43(84.3)$ & $8(15.7)$ & \multirow{2}{*}{$<0.001^{\mathrm{a}^{*}}$} \\
\hline N1 (positive) & 33 & $17(51.5)$ & $16(48.5)$ & & $13(39.4)$ & $20(60.6)$ & \\
\hline \multicolumn{8}{|l|}{ Distant metastasis } \\
\hline Absent & 82 & $29(35.4)$ & $53(64.6)$ & $0132 \mathrm{~b}$ & $56(68.3)$ & $26(31.7)$ & $0108 \mathrm{~b}$ \\
\hline Present & 2 & $2(100)$ & $0(0)$ & & $0(0)$ & $2(100)$ & \\
\hline Clinical stage & & & & & & & \\
\hline Early stages ( $\leq$ IIa) & 49 & $13(26.5)$ & $36(73.5)$ & $0020^{a^{*}}$ & $43(87.8)$ & $6(12.2)$ & $<0001^{a^{*}}$ \\
\hline Advanced stages (> IIa) & 35 & $18(51.4)$ & $17(48.6)$ & & $13(37.1)$ & $22(62.9)$ & \\
\hline Abdominal pain & & & & & & & \\
\hline Absent & 38 & $13(34.2)$ & $25(65.8)$ & $0642 \mathrm{a}$ & $22(57.9)$ & $16(42.1)$ & $0121 \mathrm{a}$ \\
\hline Present & 46 & $18(39.1)$ & $28(60.9)$ & & $34(73.9)$ & $12(26.1)$ & \\
\hline Jaundice & & & & & & & \\
\hline Absent & 69 & $23(33.3)$ & $46(66.7)$ & $0146 \mathrm{a}$ & $47(68.1)$ & $22(31.9)$ & $0.546 \mathrm{a}$ \\
\hline Present & 15 & $8(53.3)$ & $7(46.7)$ & & $9(60.0)$ & $6(40.0)$ & \\
\hline Nervous invasion & & & & & & & \\
\hline Negative & 51 & $20(39.2)$ & $31(60.8)$ & $0505 \mathrm{a}$ & $33(64.7)$ & $18(35.3)$ & 06269 \\
\hline Positive & 33 & $11(33.3)$ & $22(66.7)$ & & $23(69.7)$ & $10(30.3)$ & \\
\hline
\end{tabular}

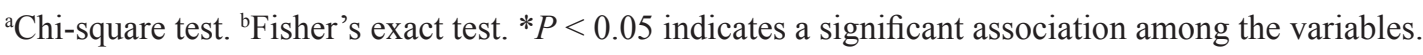


Table 5: Correlation between the clinicopathologic characteristics and HDAC3 expression $(n=84)$

\begin{tabular}{|c|c|c|c|c|c|c|c|}
\hline \multirow{2}{*}{$\begin{array}{c}\text { Clinicopathological } \\
\text { parameters }\end{array}$} & \multirow{2}{*}{ No.of patients } & \multicolumn{3}{|c|}{ Nuclear HDAC3 $(n, \%)$} & \multicolumn{3}{|c|}{ Cytoplasmic HDAC3 $(n, \%)$} \\
\hline & & Low & High & $P$-value & Low & High & $P$-value \\
\hline Cases & 84 & $38(45.2)$ & $46(54.8)$ & & $38(45.2)$ & $46(54.8)$ & \\
\hline \multicolumn{8}{|l|}{ Age (years) } \\
\hline$\leq 60$ & 39 & $19(48.7)$ & $20(51.3)$ & $0.551^{\mathrm{a}}$ & $18(46.2)$ & $21(53.8)$ & $0.875^{\mathrm{a}}$ \\
\hline$>60$ & 45 & $19(42.2)$ & $26(57.8)$ & & $20(44.4)$ & $25(55.6)$ & \\
\hline \multicolumn{8}{|l|}{ Gender } \\
\hline Male & 51 & $20(39.2)$ & $31(60.8)$ & $0.168^{a}$ & $25(49.0)$ & $26(51.0)$ & $0.387^{\mathrm{a}}$ \\
\hline Female & 33 & $18(54.5)$ & $15(45.5)$ & & $13(39.4)$ & $20(60.6)$ & \\
\hline \multicolumn{8}{|l|}{ Tumor location } \\
\hline Head, neck & 56 & $23(41.1)$ & $33(58.9)$ & $0.278^{\mathrm{a}}$ & $26(46.4)$ & $30(53.6)$ & $0.757^{\mathrm{a}}$ \\
\hline Body, tail & 28 & $15(53.6)$ & $13(46.4)$ & & $12(42.9)$ & $16(57.1)$ & \\
\hline \multicolumn{8}{|l|}{ Tumor size (cm) } \\
\hline$\leq 3$ & 25 & $10(40.0)$ & $15(60.0)$ & $0.530^{\mathrm{a}}$ & $12(48.0)$ & $13(52.0)$ & $0.741^{\mathrm{a}}$ \\
\hline$>3$ & 59 & $28(47.5)$ & $31(52.5)$ & & $26(44.1)$ & $33(55.9)$ & \\
\hline \multicolumn{8}{|l|}{ Tumor differentiation } \\
\hline Well, moderate & 57 & $27(47.4)$ & $30(52.6)$ & $0.569^{\mathrm{a}}$ & $26(45.6)$ & $31(54.4)$ & $0.920^{\mathrm{a}}$ \\
\hline Poor & 27 & $11(40.7)$ & $16(59.3)$ & & $12(44.4)$ & $15(55.6)$ & \\
\hline \multicolumn{8}{|l|}{ Invasion depth } \\
\hline $\mathrm{T} 1+\mathrm{T} 2$ & 71 & $35(49.3)$ & $36(50.7)$ & $0.081^{\mathrm{a}}$ & $29(40.8)$ & $42(59.2)$ & $0.059^{\mathrm{a}}$ \\
\hline $\mathrm{T} 3+\mathrm{T} 4$ & 13 & $3(23.1)$ & $10(76.9)$ & & $9(69.2)$ & $4(30.8)$ & \\
\hline \multicolumn{8}{|l|}{ Lymph nodes metastasis } \\
\hline N0 (negative) & 51 & $32(62.7)$ & $19(37.3)$ & $<0.001^{\text {a* }}$ & $23(45.1)$ & $28(54.9)$ & $0.974^{\mathrm{a}}$ \\
\hline N1 (positive) & 33 & $6(18.2)$ & $27(81.8)$ & & $15(45.5)$ & $18(54.5)$ & \\
\hline \multicolumn{8}{|l|}{ Distant metastasis } \\
\hline Absent & 82 & $38(46.3)$ & $44(53.7)$ & $0.499^{\mathrm{b}}$ & $36(43.9)$ & $46(56.1)$ & $0.202^{b}$ \\
\hline Present & 2 & $0(0)$ & $2(100)$ & & $2(100)$ & $0(0)$ & \\
\hline \multicolumn{8}{|l|}{ Clinical stage } \\
\hline Early stages $(\leq \mathrm{IIa})$ & 49 & $31(63.3)$ & $18(36.7)$ & $<0.001^{\text {a* }}$ & $22(44.9)$ & $27(55.1)$ & $0.941^{\mathrm{a}}$ \\
\hline Advanced stages (> IIa) & 35 & $7(20.0)$ & $28(80.0)$ & & $16(45.7)$ & $19(54.3)$ & \\
\hline \multicolumn{8}{|l|}{ Abdominal pain } \\
\hline Absent & 38 & $16(42.1)$ & $22(57.9)$ & $0.600^{\mathrm{a}}$ & $19(50.0)$ & $19(50.0)$ & $0.425^{\mathrm{a}}$ \\
\hline Present & 46 & $22(47.8)$ & $24(52.2)$ & & $19(41.3)$ & $27(58.7)$ & \\
\hline \multicolumn{8}{|l|}{ Jaundice } \\
\hline Absent & 69 & $33(47.8)$ & $36(52.2)$ & $0.307^{\mathrm{a}}$ & $31(44.9)$ & $38(55.1)$ & $0.902^{\mathrm{a}}$ \\
\hline Present & 15 & $5(33.3)$ & $10(66.7)$ & & $7(46.7)$ & $8(53.3)$ & \\
\hline \multicolumn{8}{|l|}{ Nervous invasion } \\
\hline Negative & 51 & $19(37.3)$ & $32(62.7)$ & $0.068^{\mathrm{a}}$ & $26(51.0)$ & $25(49.0)$ & $0.189^{a}$ \\
\hline Positive & 33 & $19(57.6)$ & $14(42.4)$ & & $12(36.4)$ & $21(63.6)$ & \\
\hline
\end{tabular}

${ }^{a}$ Chi-square test. ${ }^{b}$ Fisher's exact test. ${ }^{*} P<0.05$ indicates a significant association among the variables. 
Table 6: Summary of univariate and multivariate Cox regression analysis of overall survival duration in all PCs

\begin{tabular}{|c|c|c|c|c|c|c|}
\hline \multirow{2}{*}{$\begin{array}{c}\text { Clinicopathological } \\
\text { parameters }\end{array}$} & \multicolumn{3}{|c|}{ Univariate analysis } & \multicolumn{3}{|c|}{ Multivariate analysis } \\
\hline & HR & $95 \% \mathrm{CI}$ & $P$-value & HR & $95 \% \mathrm{CI}$ & $P$-value \\
\hline \multicolumn{7}{|l|}{ Membrane CDH1 } \\
\hline Low & 1 & & & & & \\
\hline High & 0.500 & $0.290-0.861$ & $0.012 *$ & & & \\
\hline \multicolumn{7}{|l|}{ Cytoplasmic CDH1 } \\
\hline Low & 1 & & & 1 & & \\
\hline High & 2.996 & $1.725-5.204$ & $<0.001 *$ & 2.204 & $1.210-4.012$ & $0.010^{*}$ \\
\hline \multicolumn{7}{|l|}{ Nuclear HDAC3 } \\
\hline Low & 1 & & & 1 & & \\
\hline High & 4.020 & $2.182-7.405$ & $<0.001^{*}$ & 3.033 & $1.572-5.852$ & $0.001 *$ \\
\hline \multicolumn{7}{|l|}{ Cytoplasmic HDAC3 } \\
\hline Low & 1 & & & & & \\
\hline High & 0.716 & $0.418-1.227$ & 0.224 & & & \\
\hline \multicolumn{7}{|l|}{ Age (years) } \\
\hline$\leq 60$ & 1 & & & & & \\
\hline$>60$ & 0.956 & $0.558-1.639$ & 0.870 & & & \\
\hline \multicolumn{7}{|l|}{ Gender } \\
\hline Male & 1 & & & & & \\
\hline Female & 0.531 & $0.295-0.957$ & $0.035^{*}$ & & & \\
\hline \multicolumn{7}{|l|}{ Tumor location } \\
\hline Head, neck & 1 & & & & & \\
\hline Body, tail & 1.189 & $0.678-2.085$ & 0.546 & & & \\
\hline \multicolumn{7}{|l|}{ Tumor size(cm) } \\
\hline$\leq 3$ & 1 & & & & & \\
\hline$>3$ & 0.797 & $0.451-1.409$ & 0.436 & & & \\
\hline \multicolumn{7}{|l|}{ Tumor differentiation } \\
\hline Well, moderate & 1 & & & 1 & & \\
\hline Poor & 2.077 & $1.192-3.620$ & $0.010^{*}$ & 2.119 & $1.210-3.711$ & $0.009 *$ \\
\hline \multicolumn{7}{|l|}{ Invasion depth } \\
\hline $\mathrm{T} 1+\mathrm{T} 2$ & 1 & & & & & \\
\hline $\mathrm{T} 3+\mathrm{T} 4$ & 0.983 & $0.463-2.088$ & 0.965 & & & \\
\hline \multicolumn{7}{|l|}{ Lymph nodes metastasis } \\
\hline N0(negative) & 1 & & & & & \\
\hline N1(positive) & 2.060 & $1.196-3.546$ & $0.009^{*}$ & & & \\
\hline \multicolumn{7}{|l|}{ Distant metastasis } \\
\hline Absent & 1 & & & & & \\
\hline Present & 2.372 & $0.574-9.798$ & 0.233 & & & \\
\hline \multicolumn{7}{|l|}{ Clinical stage } \\
\hline Early stages $(\leq \mathrm{IIa})$ & 1 & & & & & \\
\hline Advanced stages (> IIa) & 2.230 & $1.294-3.845$ & $0.004 *$ & & & \\
\hline
\end{tabular}




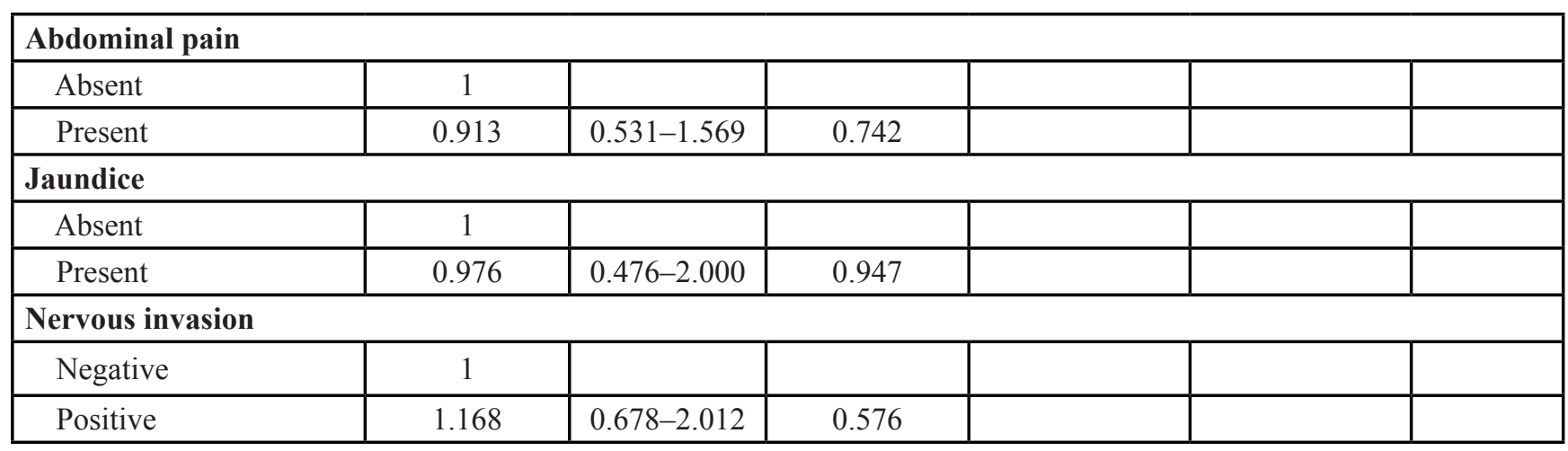

HR hazard ratio, 95\% CI 95\% confidence interval.

Univariate analysis also indicated that gender, tumor differentiation, lymph node metastasis, and clinical stage correlated with patient survival $(P=0.035$, $P=0.010, P=0.009$, and $P=0.004$, respectively). Multivariate analysis shows that cytoplasmic $\mathrm{CDH} 1$ expression, nuclear HDAC3 expression, and tumor differentiation were independent prognostic factors for PC patients (Table 6). Membrane CDH1 expression, gender, lymph node metastasis, and clinical stage were not associated with survival (Table 6). To further investigate the association of survival time with cytoplasmic CDH1 and nuclear HDAC3 expression, a final concomitant model was constructed. As shown in Figure 2E, the log-rank test showed that high co-expression of these two proteins correlated with shorter survival time of PC patients $(P<0.001)$. The cumulative proportion of 1-year survival was only $12 \%(95 \%$ CI, $0.002-0.238)$ in the high co-expression group and $64 \%(95 \% \mathrm{CI}, 0.522-0.758)$ in other combination groups (Table 7). Moreover, Spearman correlation analysis revealed a positive correlation between the high co-expression group and lymph nodes metastasis, clinical stage $(r=0.436$ and $r=0.506$, respectively, Supplementary Table S5).

\section{DISCUSSION}

Cellular functions are dictated by protein activity and content. There are numerous strategies to regulate proteins varying from modulating gene expression to post-translational modifications to control of protein localization [15]. Numerous studies demonstrate functionally relevant subcellular translocation of specific individual proteins [16]. For example, $\beta$-catenin is found at multiple subcellular localizations, including at cell junctions, where it stabilizes cell-cell contacts; in the cytoplasm, where $\beta$-catenin levels are controlled by protein stability regulating processes; and in the nucleus, where $\beta$-catenin is involved in transcriptional regulation and chromatin interactions $[17,18]$. Moreover, $\beta$-catenin nuclear import and accumulation drives tumor formation and correlates with clinical tumor grade [19]. Another example is BRCA1, whose prognostic significance varies with its subcellular distribution. Nuclear detection of the protein is associated with a worse prognosis, while cytoplasmic localization predicts lower probability of recurrence due to fewer lymph node metastases [20].

Dysfunction of the CDH1-mediated cell adhesion system plays an important role in pancreatic tumor progression to invasive, metastatic carcinoma [21, 22]. Epigenetic modifications contribute to loss of $\mathrm{CDH} 1$ expression [23, 24]. Yao R et al [25] found that HDAC3 binds the $\mathrm{CDH} 1$ promoter, resulting in reduced local histone acetylation and $\mathrm{CDH} 1$ transcriptional repression [25]. We previously revealed that HDAC3 is overexpressed in PC tissue, and increased HDAC3 can promote malignant tumor phenotypes [12]. Moreover, Hayashi A et al [13] found that HDAC3 was inversely correlated with $\mathrm{CDH} 1$ expression in ovarian carcinoma. In this study, we determined the expression pattern of CDH1 and HDAC3 proteins in PC tissues, and the clinicopathological and prognostic value of those subcellular localizations.

High-throughput TMA was employed to perform our research. First, we found that CDH1 was predominantly found on the cell membrane and in the cytoplasm, while HDAC3 localized to cell nucleus and cytoplasm. Further analysis revealed that the cell membrane CDH1 was greatly reduced in $\mathrm{PC}$ tissues compared to noncancerous epithelia, whereas nuclear HDAC3 was abnormally upregulated. Furthermore, there was an inverse association between these two proteins in PC tissues, consistent with recent reports on ovarian carcinoma [13].

It is worth noting that abnormal cytoplasmic $\mathrm{CDH} 1$ in $\mathrm{PC}$ tissues, and higher cytoplasmic $\mathrm{CDH} 1$ expression were associated with more aggressive tumorassociated variables, including lymph node metastasis and advanced clinical stage. Moreover, PC patients with high cytoplasmic CDH1 expression had shorter OS than the low-expression group. In contrast, reduced membrane $\mathrm{CDH} 1$ correlated with lymph node metastasis, advanced clinical stage, and shorter survival time. Multivariate analyses demonstrate that cytoplasmic but not membrane CDH1 expression was an independent prognostic factor for PC. Previously, Deeb G et al [26] found that cytoplasmic staining of $\mathrm{CDH} 1$ in lung cancer 
tissues correlates with shorter patient survival. Ito $\mathrm{K}$ et al [27] revealed that CDH1 cytoplasmic staining may be due to $\mathrm{CDH} 1$ proteolytic cleavage by a membranebound metalloprotease, yielding a soluble form. Although nuclear staining of CDH1 protein has been associated with skin Merkel cell carcinomas [28], we did not observe nuclear CDH1 in our PC patient cohort. Taken together, cytoplasmic $\mathrm{CDH} 1$ expression appears to represent altered protein localization related to $\mathrm{PC}$ tumorigenicity.

HDAC3 is the only class I HDAC found in the nucleus, cytoplasm, and plasma membrane [29, 30]. Previous studies focused on its function as an epigenetic modifier, repressing transcription through histone deacetylation $[10,31,32]$. Few studies have investigated the prognostic role of altered HDAC3 localization in PC. In this study, we found HDAC3 in the cytoplasm and nucleus of tumor cells, but not on the plasma membrane. Higher nuclear HDAC3 expression was observed in $\mathrm{PC}$ relative to adjacent normal tissues, while cytoplasmic expression of HDAC3 was indistinguishable. Cytoplasmic staining of HDAC3 was not associated with any clinicopathologic features or survival in PC patients. In contrast, increased nuclear HDAC3 expression was strongly associated with $\mathrm{N}$ classification and advanced clinical stage. For example, nuclear HDAC3 expression was detected in $80.0 \%$ of patients with high tumor grade ( $>$ IIa), but only $36.7 \%$ in the low tumor grade group $(\leq \mathrm{IIa})$, suggesting that nuclear HDAC3 plays an important role in tumor progression in PC patients. Univariate analysis showed that nuclear HDAC3 in PC was associated with patients' OS. Higher nuclear HDAC3 correlates with worse prognosis. Furthermore, according to multivariate analysis, overexpression of nuclear HDAC3 has independent prognostic significance for PC. It is of particular note that high nuclear HDAC3 expression was positively associated with increased cytoplasmic CDH1. High co-expression of these two proteins correlated with shorter patient survival, with a cumulative 1-year survival of $12 \%(95 \%$ CI, $0.002-0.238)$ compared to that of $64 \%$ (95\% CI, 0.522-0.758) in other expression levels group. Escaffit $\mathrm{F}$ et al [33] reported that nuclear localization of HDAC3 decreases the efficiency of apoptosis induction, and HDAC3 cytoplasmic relocalization is important for the apoptotic process.

We speculate that first, pancreatic tumor cells may have escaped apoptosis, at least in part, through HDAC3 overexpression in cell nucleus. Secondly, high concentrations of nuclear HDAC3 may directly inhibit CDH1 promoters, leading to reduced $\mathrm{CDH} 1$ cell membrane expression. Additionally, nuclear HDAC3 expression may upregulate membrane-bound metalloprotease expression through epigenetic modification of the associated target gene, leading to increased cytoplasmic CDH1. Together, our findings strongly indicate that nuclear HDAC3 upregulation is crucial for the aggressive behaviors and worse prognosis
A

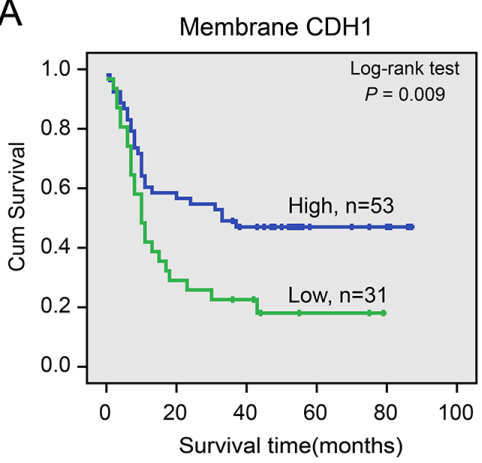

$\mathrm{D}$

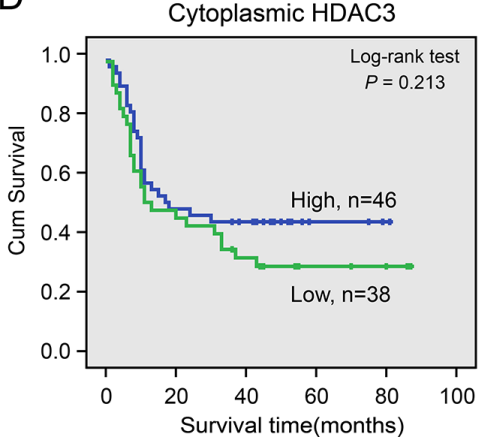

B

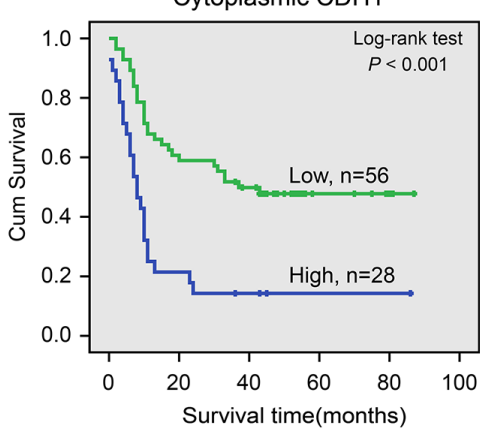

E

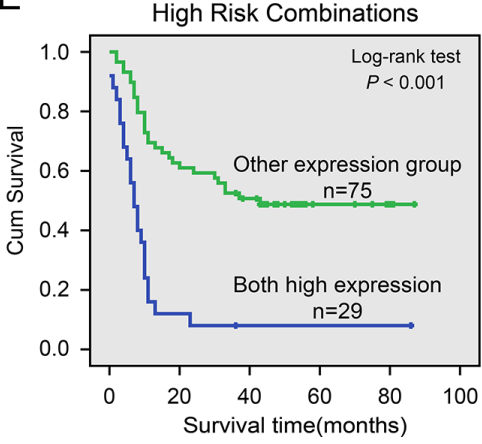

C

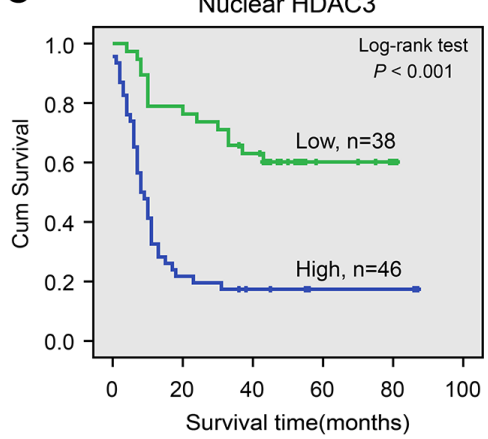

$\Pi$ Other expression group

Nuclear HDAC3(Low), Cytoplasmic CDH1(Low) Nuclear HDAC3(High), Cytoplasmic CDH1(Low) Nuclear HDAC3(Low), Cytoplasmic CDH1(High)

$\Pi$ Both high expression

Nuclear HDAC3(High), Cytoplasmic CDH1(High)

Figure 2: Cumulative kaplan-meier overall survival curves of 84 PC patients segmented by CDH1 (A), membrane CDH1; (B), cytoplasmic CDH1), HDAC3 (C), nuclear HDAC3; (D), cytoplasmic HDAC3), and high-risk combination group (cytoplasmic CDH1 and nuclear HDAC3 combinations) (E). $P$-values were calculated by the log-rank test. 
Table 7: Comparisons with cumulative 1-year survival rate between different groups

Variables
\begin{tabular}{|l|c|c|}
\hline Membrane CDH1 & & 95\% CI \\
\hline Low & $32 \%$ & $0.163-0.477$ \\
\hline High & $58 \%$ & $0.443-0.717$ \\
\hline Cytoplasmic CDH1 & & \\
\hline Low & $63 \%$ & $0.512-0.748$ \\
\hline High & $21 \%$ & $0.053-0.367$ \\
\hline Nuclear HDAC3 & & \\
\hline Low & $79 \%$ & $0.653-0.927$ \\
\hline High & $24 \%$ & $0.122-0.358$ \\
\hline Cytoplasmic HDAC3 & & $0.313-0.627$ \\
\hline Low & $47 \%$ & $0.363-0.637$ \\
\hline High & $50 \%$ & \\
\hline High risk combinations & & $0.002-0.238$ \\
\hline Both high expression & $12 \%$ & $0.522-0.758$ \\
\hline Other expression group & $64 \%$ & \\
\hline
\end{tabular}

$95 \%$ CI, 95\% confidence interval.

of PC patients, which suggest that HDAC3 may be an effective therapeutic target. Unfortunately, clinical data for HDAC inhibitors (HDACIs) are inadequate, because few studies have included patients with PC and few PC patients entered the HDACIs phase II/III trials that did [34]. More high quality clinical trials recruiting candidates with $\mathrm{PC}$ are required to determine the efficacy of these therapies. Selective HDACIs, potentially targeting HDAC3, may yield more potent efficacy and fewer side effects than pan-HDACIs.

In summary, these data strongly suggest the importance of nuclear HDAC3 and cytoplasmic $\mathrm{CDH} 1$ in the progression and clinical outcome of human PC. These markers provide strong candidates for targeted therapy of PC patients. Larger prospective studies could further validate these findings.

\section{MATERIALS AND METHODS}

\section{Patients and tissue samples}

This study was approved by the Ethics and Research Committees of Shanghai General Hospital, Shanghai Jiao Tong University School of Medicine, and was conducted in accordance with the Declaration of Helsinki Principles. TMAs containing 90 PC tissues and corresponding nontumor tissues were purchased from ShGnghGi Outdo Biotech Company (China). The TMAs contained welldocumented clinicopathological information, including patients' age, sex, tumor size and location, tumor differentiation, invasion depth, lymph node metastasis, distant metastasis, clinical stage, abdominal pain, jaundice, nervous invasion, and follow-up data (ended in December, 2011). Six patients were excluded due to lack of completed clinical and follow-up data. In total, 84 patients were included, 51 males and 33 females, with a median age of 62 years old (ranging from 38 to 85 years old). The overall survival time ranged from 0 to 87 months, with a median of 15 months. Detailed information can be found in Table 8 .

\section{Immunohistochemistry}

Immunohistochemistry was performed based on the standard streptavidin-peroxidase (S-P) method (Zymed, San Francisco, CA). After deparaffinization and rehydration, TMA sections were subjected to high pressure for antigen retrieval for 5 minutes. Endogenous peroxidase activity was blocked using $100 \mu \mathrm{L}$ of peroxidase block for $10 \mathrm{~min}$. The slides were subsequently incubated overnight at $4{ }^{\circ} \mathrm{C}$ with primary antibodies as follows: $\mathrm{CDH} 1$ (dilution 1:300, BD Biosciences), HDAC3 (dilution 1:500, Abcam). After washing in $1 \times$ phosphate buffered saline (PBS), the sections were incubated with biotinylated secondary antibodies (Zymed, San Francisco, CA) for $30 \mathrm{~min}$ at room temperature, followed by incubation with streptavidin horseradish peroxidase complex. Finally, sections were incubated with DAB for 2 min. Positive controls were used in each experiment following supplier's instructions. Negative controls applying appropriate $\operatorname{IgG}$ to replace 
Table 8: Detailed clinical information of patients with PC

\begin{tabular}{|c|c|c|}
\hline \multicolumn{2}{|l|}{ Characteristics } & Number \\
\hline \multicolumn{2}{|c|}{ Overall survival median (range, months) } & $15(0-87)$ \\
\hline \multicolumn{2}{|l|}{ Age median (range, years) } & $62(38-85)$ \\
\hline \multirow{2}{*}{ Tumor location } & Head, neck & 56 \\
\hline & Body, tail & 28 \\
\hline \multirow{2}{*}{ Tumor size $(\mathrm{cm})$} & $\leq 3$ & 25 \\
\hline & $>3$ & 59 \\
\hline \multirow{2}{*}{ Tumor differentiation } & Well, moderate & 57 \\
\hline & Poor & 27 \\
\hline \multirow{2}{*}{ Invasion depth } & $\mathrm{T} 1+\mathrm{T} 2$ & 71 \\
\hline & $\mathrm{T} 3+\mathrm{T} 4$ & 13 \\
\hline \multirow{2}{*}{ Lymph nodes metastasis } & N0 (negative) & 51 \\
\hline & N1 (positive) & 33 \\
\hline \multirow{2}{*}{ Distant metastasis } & Absent & 82 \\
\hline & Present & 2 \\
\hline \multirow{2}{*}{ Clinical stage } & Early stages $(\leq$ IIa $)$ & 49 \\
\hline & Advanced stages (> IIa) & 35 \\
\hline \multirow{2}{*}{ Abdominal pain } & Absent & 38 \\
\hline & Present & 46 \\
\hline \multirow{2}{*}{ Jaundice } & Absent & 69 \\
\hline & Present & 15 \\
\hline \multirow{2}{*}{ Nervous invasion } & Negative & 51 \\
\hline & Positive & 33 \\
\hline
\end{tabular}

primary antibody were also run in each experiment (Supplementary Figure 1A, 1B).

\section{Scoring of immunohistochemistry}

A double-blind method, carried out independently by two investigators without access to the patients' clinical and pathological features, was used to analyze immunohistochemistry results. Five visual fields from different areas of each specimen were chosen at random for the immunohistochemistry evaluation. HDAC3 and $\mathrm{CDH} 1$ expression was scored according to staining intensity and the percentage of positive cells as previously described [35]. The percentage of positive cells was scored as follows: $0 \%(0), 1 \%-10 \%(1), 11 \%-50 \%(2)$ and $51 \%-100 \%$ (3). Staining intensity was scored as follows: no staining (0), week (1), moderate (2), and strong (3). Comprehensive score $=$ staining percentage $\times$ intensity . CDH1 or HDAC3 expression was classified as follows: < 6 low expression, $\geq 6$ high expression.

\section{Statistical analysis}

All statistical analyses were carried out using the SPSS 13.0 software. The $\chi^{2}$ test and Fisher's exact test were used to analyze the correlation between the clinicopathologic characteristics and CDH1 and HDAC3 expression as appropriate. Overall survival (OS) was defined as the interval from date of diagnosis until death from any cause. Data were censored for living patients and patients lost between follow-ups. The OS was estimated using the Kaplan-Meier method and compared using the log-rank test. Significant variables were further analyzed by multivariate analysis to test for independent prognosis. Bivariate correlations between variable factors were calculated by Spearman rank correlation coefficients. $P$-values $<0.05$ were considered statistically significant.

\section{ACKNOWLEDGMENTS AND FUNDING}

This study was supported in part by the National Natural Science Foundation of China (grant NO. $81502017,81502018,81572315,81101846,81171887$ and 91229117), by Research Grant from Shanghai Hospital Development Center (SHDC12014128), by National Key Clinical Discipline-Oncology, and by Songjiang Liandong Program(0702N14002).

\section{CONFLICTS OF INTEREST}

The authors declare no conflicts of interests. 


\section{REFERENCES}

1. Torre LA, Bray F, Siegel RL, Ferlay J, Lortet-Tieulent J, Jemal A. Global cancer statistics, 2012. CA Cancer J Clin 2015;65:87-108.

2. Rahib L, Smith BD, Aizenberg R, Rosenzweig AB, Fleshman JM, Matrisian LM. Projecting cancer incidence and deaths to 2030: the unexpected burden of thyroid, liver, and pancreas cancers in the United States. Cancer Res 2014;74:2913-2921.

3. Hidalgo M. Pancreatic cancer. N Engl J Med 2010;362:1605-1617.

4. Chames P, Kerfelec B, Baty D. Therapeutic antibodies for the treatment of pancreatic cancer. ScientificWorldJournal 2010;10:1107-1120.

5. Le N, Sund M, Vinci A. Prognostic and predictive markers in pancreatic adenocarcinoma. Dig Liver Dis 2015.

6. Becker KF, Atkinson MJ, Reich U, Becker I, Nekarda H, Siewert JR, Hofler H. E-cadherin gene mutations provide clues to diffuse type gastric carcinomas. Cancer Res 1994;54:3845-3852.

7. Grady WM, Willis J, Guilford PJ, Dunbier AK, Toro TT, Lynch H, Wiesner G, Ferguson K, Eng C, Park JG, Kim SJ, Markowitz S. Methylation of the $\mathrm{CDH} 1$ promoter as the second genetic hit in hereditary diffuse gastric cancer. Nat Genet 2000;26:16-17.

8. Shibata T, Hirohashi S. [E-cadherin cell adhesion system in human cancer. Seikagaku 2006;78:647-656.

9. Liu X, Wang JH, Li S, Li LL, Huang M, Zhang YH, Liu Y, Yang YT, Ding R, Ke YQ. Histone deacetylase 3 expression correlates with vasculogenic mimicry through the phosphoinositide3-kinase / ERK-MMP-laminin5gamma2 signaling pathway. Cancer Sci 2015;106:857-866.

10. Ma Y, Yue Y, Pan M, Sun J, Chu J, Lin X, Xu W, Feng L, Chen Y, Chen D, Shin VY, Wang X, Jin H. Histone deacetylase 3 inhibits new tumor suppressor gene DTWD1 in gastric cancer. Am J Cancer Res 2015;5:663-673.

11. Pilarsky C, Wenzig M, Specht T, Saeger HD, Grutzmann R. Identification and validation of commonly overexpressed genes in solid tumors by comparison of microarray data. Neoplasia 2004;6:744-750.

12. Jiao F, Hu H, Yuan C, Jin Z, Guo Z, Wang L, Wang L. Histone deacetylase 3 promotes pancreatic cancer cell proliferation, invasion and increases drug-resistance through histone modification of P27, P53 and Bax. Int J Oncol 2014;45:1523-1530.

13. Hayashi A, Horiuchi A, Kikuchi N, Hayashi T, Fuseya C, Suzuki A, Konishi I, Shiozawa T. Type-specific roles of histone deacetylase (HDAC) overexpression in ovarian carcinoma: HDAC1 enhances cell proliferation and HDAC3 stimulates cell migration with downregulation of E-cadherin. Int J Cancer 2010;127:1332-1346.
14. Hayashi A, Horiuchi A, Kikuchi N, Hayashi T, Fuseya C, Suzuki A, Konishi I, Shiozawa T. Type-specific roles of histone deacetylase (HDAC) overexpression in ovarian carcinoma: HDAC1 enhances cell proliferation and HDAC3 stimulates cell migration with downregulation of E-cadherin. Int J Cancer 2010;127:1332-1346.

15. Bauer NC, Doetsch PW, Corbett AH. Mechanisms Regulating Protein Localization. Traffic 2015;16:1039-1061.

16. He H, Lee MC, Zheng LL, Zheng L, Luo Y. Integration of the metabolic/redox state, histone gene switching, DNA replication and S-phase progression by moonlighting metabolic enzymes. Biosci Rep 2013;33:e18.

17. Voronkov A, Krauss S. Wnt/beta-catenin signaling and small molecule inhibitors. Curr Pharm Des 2013;19:634-664.

18. Prakash S, Swaminathan U. beta catenin in health: A review. J Oral Maxillofac Pathol 2015;19:230-238.

19. Jamieson C, Sharma M, Henderson BR. Targeting the betacatenin nuclear transport pathway in cancer. Semin Cancer Biol 2014;27:20-29.

20. Mylona E, Melissaris S, Nomikos A, Theohari I, Giannopoulou I, Tzelepis K, Nakopoulou L. Effect of BRCA1 immunohistochemical localizations on prognosis of patients with sporadic breast carcinomas. Pathol Res Pract 2014;210:533-540.

21. Beuran M, Negoi I, Paun S, Ion AD, Bleotu C, Negoi RI, Hostiuc S. The epithelial to mesenchymal transition in pancreatic cancer: A systematic review. Pancreatology 2015;15:217-225.

22. Nagathihalli NS, Merchant NB. Src-mediated regulation of E-cadherin and EMT in pancreatic cancer. Front Biosci (Landmark Ed) 2012;17:2059-2069.

23. Peinado H, Ballestar E, Esteller M, Cano A. Snail mediates E-cadherin repression by the recruitment of the Sin3A/ histone deacetylase 1 (HDAC1)/HDAC2 complex. Mol Cell Biol 2004;24:306-319.

24. Dong C, Wu Y, Yao J, Wang Y, Yu Y, Rychahou PG, Evers BM, Zhou BP. G9a interacts with Snail and is critical for Snailmediated E-cadherin repression in human breast cancer. J Clin Invest 2012;122:1469-1486.

25. Yao R, Jiang H, Ma Y, Wang L, Wang L, Du J, Hou P, Gao Y, Zhao L, Wang G, Zhang Y, Liu DX, Huang B, et al. PRMT7 induces epithelial-to-mesenchymal transition and promotes metastasis in breast cancer. Cancer Res 2014;74:5656-5667.

26. Deeb G, Wang J, Ramnath N, Slocum HK, Wiseman S, Beck A, Tan D. Altered E-cadherin and epidermal growth factor receptor expressions are associated with patient survival in lung cancer: a study utilizing high-density tissue microarray and immunohistochemistry. Mod Pathol 2004; 17:430-439.

27. Ito K, Okamoto I, Araki N, Kawano Y, Nakao M, Fujiyama S, Tomita K, Mimori T, Saya H. Calcium influx triggers the 
sequential proteolysis of extracellular and cytoplasmic domains of E-cadherin, leading to loss of beta-catenin from cell-cell contacts. Oncogene 1999;18:7080-7090.

28. Han AC, Soler AP, Tang CK, Knudsen KA, Salazar H. Nuclear localization of E-cadherin expression in Merkel cell carcinoma. Arch Pathol Lab Med 2000;124:1147-1151.

29. Gao Z, He Q, Peng B, Chiao PJ, Ye J. Regulation of nuclear translocation of HDAC3 by IkappaBalpha is required for tumor necrosis factor inhibition of peroxisome proliferatoractivated receptor gamma function. $\mathrm{J}$ Biol Chem 2006;281:4540-4547.

30. Longworth MS, Laimins LA. Histone deacetylase 3 localizes to the plasma membrane and is a substrate of Src. Oncogene 2006;25:4495-4500.

31. Barneda-Zahonero B, Parra M. Histone deacetylases and cancer. Mol Oncol 2012;6:579-589.

32. El-Hage P, Petitalot A, Monsoro-Burq AH, Maczkowiak F, Driouch K, Formstecher E, Camonis J, Sabbah M, Bieche I,
Lidereau R, Lallemand F. The Tumor-Suppressor WWOX and HDAC3 Inhibit the Transcriptional Activity of the betaCatenin Coactivator BCL9-2 in Breast Cancer Cells. Mol Cancer Res 2015;13:902-912.

33. Escaffit F, Vaute O, Chevillard-Briet M, Segui B, Takami Y, Nakayama T, Trouche D. Cleavage and cytoplasmic relocalization of histone deacetylase 3 are important for apoptosis progression. Mol Cell Biol 2007;27:554-567.

34. Koutsounas I, Giaginis C, Theocharis S. Histone deacetylase inhibitors and pancreatic cancer: are there any promising clinical trials? World J Gastroenterol 2013;19:1173-1181.

35. Luo W, Fang W, Li S, Yao K. Aberrant expression of nuclear vimentin and related epithelial-mesenchymal transition markers in nasopharyngeal carcinoma. Int $\mathrm{J}$ Cancer 2012;131:1863-1873. 